\title{
Aceros Bainíticos y la Influencia del Boro sobre sus Propiedades Mecánicas - Revisión Bibliográfica
}

\section{Influence of Boron in the mechanical properties of Bainitic steels}

\author{
Karol Felipe Rodríguez Galeano \\ Ingeniero Mecánico, Universidad Nacional de Colombia \\ Estudiante de Maestría en Ingeniería - materiales y procesos \\ Universidad Nacional de Colombia \\ Bogotá, Colombia. \\ kfrodriguezg@unal.edu.co
}

\begin{abstract}
Resumen- En las últimas décadas se han hecho importantes desarrollos en equipos industriales de calentamiento y enfriamiento como hornos y muflas, entre otros, que los hacen muy estables y permiten hacer incrementos y decrementos de temperatura de una forma muy controlada y precisa; por esto se ha permitido hacer estudios sobre materiales que necesitan de ciertos parámetros para adquirirlos, mediante procesos térmicos y mecánicos combinados como es el caso de los aceros bainíticos. Hoy se investiga sobre este tipo de aceros, variándole porcentajes de aleantes como lo es el boro y observar cómo varían sus propiedades mecánicas con cada cambio, lo que permite caracterizarlos y dar una fuerte base a las industrias para que utilicen nuevos materiales que tengan menos procesos de fabricación, para que sean más económicos y menos hostiles con el medio ambiente. En el presente artículo se caracteriza la microestructura bainitica y se tienen en cuenta los cambios de distintas propiedades que son de suma relevancia industrial respecto al cambio de la cantidad de Boro presente, algunas de estas propiedades son el esfuerzo de fluencia, el esfuerzo último a la tensión, la deformación de ingeniería cuando estos materiales son sometidos a tensión y una comparación con los aceros perliticos, los cuales representan una parte importante de aplicaciones industriales.
\end{abstract}

Palabras Clave- aceros bainíticos, boro, propiedades mecánicas.

ABSTRACT- In the last decades there have been important developments in industrial equipment of heating and cooling such as are furnaces, muffles, among others, making them very stable and allowing to make temperature increases and decreases in a controlled and precise way; which is why they studies about parameters needed to acquire special materials have allowed, by combined thermal and mechanical processes such as the bainitic steels. Today it continues to research this type of steel, changing alloys percentages and watching how they can make mechanical properties vary, thus allowing to characterize and provide a strong basis for industries using new materials that have less manufacturing processes, and being more economical and less hostile to the environment. In this article bainite microstructure is characterized and takes into account the changes of properties that are of great industrial importance regarding the change of the amount of boron present, some of these properties are the yield stress, ultimate stress to stress, the engineering strain when these materials are subjected to strain and a comparison with pearlitic steels, which represent a significant part of industrial applications for steel.

Keywords- bainitic steels, boron, mechanical properties.

\section{INTRODUCCIÓN}

Los aceros bainíticos representan hoy en día una gran opción para remplazar en varias aplicaciones a los aceros de alta aleación, debido a su relativo bajo costo y a que presentan mejores propiedades mecánicas como lo es la tenacidad y la dureza, aparte de esto tienen una estructura más fina que los aceros perlíticos a base de partículas diminutas de Cementita en una matriz ferrítica por este motivo exhiben una interesante combinación de resistencia y ductilidad. [1]. Sin embargo, en muchos lugares se obtienen estos aceros con combinaciones de ferroaleantes que, a su vez, pueden tener boro presente, el cual permite aumentar sensiblemente la templabilidad de los aceros al carbono de baja aleación. [2].

Existe gran variedad de aceros bainíticos: de alto carbono, con alta soldabilidad; aceros 
de alta resistencia, que compiten con los templados y revenidos; aceros resistentes a altas temperaturas, usados en las centrales nucleares; aceros inoculados en los cuales, la bainita nuclea al interior de los granos de austenita produciéndose una microestructura resistente a la propagación de grietas y otros. La microestructura de los aceros de alta resistencia está compuesta de ferrita bainítica, martensita y austenita retenida. Su templabilidad puede ser mejorada al añadir a su composición de manganeso, cromo y níquel. Generalmente presentan una gran concentración en silicio que evita la formación de cementíta. [3]. Este tipo de aceros no solo tiene aplicaciones en usos científicos o aeronáuticos, Los aceros bainíticos con bajo contenido de carbono y series de manganeso han sido ampliamente usados en muchos campos de la industria para reducir costos y obtener entre otros un fácil procesamiento, alta templabilidad y buenas propiedades mecánicas [34][35], haciéndolos óptimos para industrias que tengan que ver con piezas sometidas a desgaste y en construcción de rieles para medios de transporte ferroviario.

\section{BAINITA}

Los aceros y las fundiciones nodulares, en estado bainítico, son familias de aleaciones de $\mathrm{Fe}-\mathrm{C}$ que permiten obtener una amplia gama de propiedades mecánicas. Este hecho ha incentivado el estudio de la reacción bainítica y sus aplicaciones dirigidas a la sustitución de aceros altamente aleados para la fabricación de componentes de maquinaria en los que se requiere alta resistencia a la tracción y capacidad de absorber energía de impacto. [4]. Adicionalmente, las microestructuras bainiticas han encontrado aplicación en sistemas donde se requiere resistencia al desgaste, ya sea asociado a fenómenos de rodadura y deslizamiento, como es el caso del contacto rueda-riel. [5].

La microestructura de la bainita consiste en listones o placas nucleados en los límites de grano austenítico, al igual que en las transformaciones de fase difusivas. El paquete bainítico está conformado por listones o placas (subunidades micro-estructurales) paralelos como se puede observar en la Fig. 1.
Fig. 1. EVOLUCIÓN DE UNA PLUMA BAINÍTICA EN FUNCIÓN DEL TIEMPO. [4]

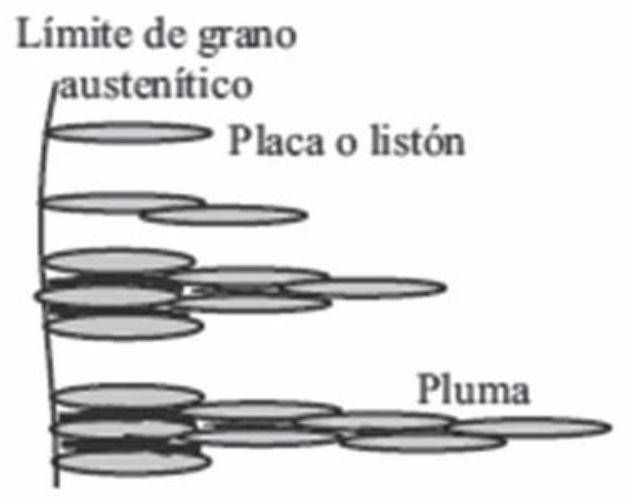

El estudio de la transformación bainítica se ha visto limitado algunas veces debido al tamaño de las unidades micro-estructurales, ya que la placa observada en el microscopio óptico es en realidad una pluma bainítica formada por varias subunidades; este tamaño reducido se debe principalmente a las temperaturas de transformación, que afectan la difusión del carbono, y a la precipitación de películas de cementíta en los límites entre las subunidades, que impiden su crecimiento. [6].

La complejidad de la microestructura bainítica ha dado lugar a la existencia de multitud de terminologías para representar su morfología, lo que ha conducido a cierta confusión. [7]. Se denomina ferrita bainítica $\left(\alpha_{\mathrm{b}}\right)$ a la fase ferrita cuando se encuentra en forma de subunidad de bainita. Las subunidades de ferrita bainítica pueden contener partículas de cementíta en su interior, que da lugar a la denominada bainita inferior. $\mathrm{Si}$, por el contrario, la ferrita bainítica está libre de partículas de cementíta, la microestructura bainítica se denomina bainita superior. La bainita superior se forma a mayores temperaturas que la inferior. [8].

Fig. 2. ESQUEMA DE BAINITA SUPERIOR Y BAINITA INFERIOR [8]

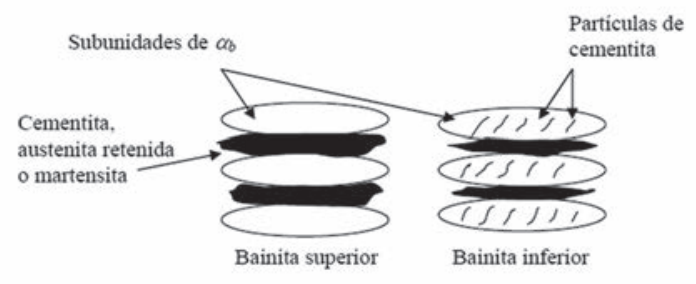


Fig. 3. A) MICROGRAFÍA DE LUZ QUE MUESTRA POLEAS DE BAINITA INFERIOR EN UNA ALEACIÓN FE-0.3C-4CR WT\% PARCIALMENTE TRANSFOR$\operatorname{MADA}\left(395^{\circ} \mathrm{C}\right)$. EL GRABADO LUMINOSO MUESTRA UNA MATRIZ DE FASE MARTENSÍTICA

B) CORRESPONDIENTE A UN MICROSCOPIO DE TRANSMISIÓN DE ELECTRONES QUE MUESTRA SUBUNIDADES DE BAINITA INFERIOR [1]
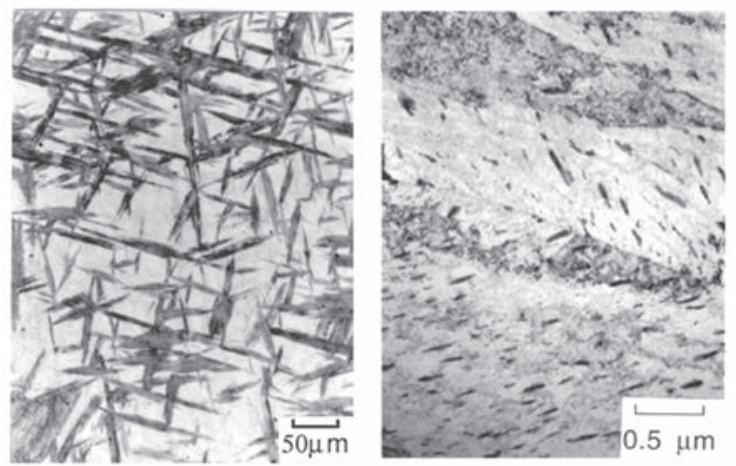

La morfología de placa tiende a formarse a bajas temperaturas y a altas concentraciones de carbono y, en general, cuando la austenita madre presenta alta resistencia. [1]. De igual manera, el espesor de placa es un importante parámetro en la caracterización de la ferrita bainítica, pues su afinamiento da lugar a un aumento simultáneo de resistencia y tenacidad en el material. Es sabido que el espesor de placa disminuye con la temperatura de transformación. [10]-[11]. En particular, Parker [12] ha encontrado la siguiente dependencia:

$$
\mathrm{u}_{\mathrm{e}}=0,2 * 10^{-6}\left(\frac{T-528}{150}\right)
$$

Donde $u_{e}$ es el espesor de la subunidad de la ferrita bainítica y $T$ la temperatura en K. En los estudios de Singh y Bhadeshia, es la resistencia de la austenita la principal responsable del valor del espesor de placa de la ferrita bainítica $\alpha b$. [13].

Para poder obtener la transformación bainítica, se necesita de ciertas condiciones termodinámicas, desde el punto de vista teórico y de acuerdo con Bhadeshia [14], las condiciones termodinámicas que se deben cumplir para la transformación bainítica son:

Nucleación

$$
\Delta G_{m}<G_{N}
$$

Crecimiento

$$
\Delta G^{y \rightarrow \alpha}<-G_{S B}
$$

La función GN, también denominada función universal de nucleación, debido a que depende de la temperatura y no de la composición química, en los aceros de baja aleación tiene la forma [15].

$$
G_{N}=3.546 T-3499.4
$$

T es la temperatura en grados Kelvin

La bainita es un micro constituyente que se obtiene mediante procesos de transformación isotérmica, generalmente a temperaturas entre 200 y $300^{\circ} \mathrm{C}$. Sin embargo, para acelerar la transformación de este elemento se adicionan aleantes como el aluminio y el cobalto [31], en concentraciones inferiores al $2 \%$ dentro de la composición química del acero [32]-[33].

Por encontrarse entre una transformación difusional (ferrita/perlita) y otra adifusional (martensíta), la bainita conserva características de ambas. Así su nucleación tiene lugar bajo condiciones de para equilibrio, solo el carbono difunde, y el crecimiento es completamente adifusional, tipo martensítico. Cuando un núcleo crece, lo hace con una morfología acicular, para poder acomodar la deformación plástica inducida en la austenita, una vez que el crecimiento de una aguja/placa de ferrita bainítica termina, el exceso de Carbono que pueda tener se difunde a la austenita vecina, de forma tal que la siguiente placa ha de crecer a partir de una austenita enriquecida en Carbono. Esto ocurrirá siempre y cuando la concentración de carbono no supere un valor límite teórico denominado curva [3]. Que representa el límite de concentración de carbono de la austenita por encima del cual no puede tener lugar una transformación adifusional. [8].

Debido a la saturación de carbono en la austenita, existe posibilidad de saturación de cementíta desde la austenita enriquecida. Sin embargo, esta precipitación característica de la bainita común, resulta perjudicial por su efecto fragilizante y se ha descubierto que puede ser evitada por la adición de Si en cantidades adecuadas, por ejemplo 1.5\% Si [1]-[16]. Para la aplicación óptima de los aceros bainíticos, estos se encuentren libres de carburos con el fin de obtener una microestructura que contenga placas de ferrita y austenita retenida, que elimina comportamientos no deseables a temperatura ambiente como lo sería el fragilizante dado por la presencia de cementíta. Esta 
precipitación de cementíta durante la transformación bainítica puede evitarse al alear el acero con silicio en cantidades de un 1,5\% aproximadamente. El silicio presenta muy baja solubilidad en la cementíta y retrasa el crecimiento de la misma en la austenita residual tras la formación de bainita. [17]. El carbono expulsado como consecuencia de la formación de la ferrita bainítica enriquecerá la austenita residual, produciéndose así su estabilización hasta la temperatura ambiente. El resultado es una microestructura formada por placas muy finas de ferrita bainítica separadas por regiones de austenita enriquecidas en carbono. Una microestructura de estas características es ideal desde muchos puntos de vista; en primer lugar, el acero tendrá una alta resistencia a la fractura por clivaje. Por otro lado, existe la posibilidad de mejorar, simultáneamente, la resistencia y la tenacidad debido al tamaño de grano ultrafino de las placas de ferrita bainítica y al efecto de transformación inducida por deformación plástica. Sin embargo, a pesar de todas estas ventajas, la microestructura de ferrita bainítica-austenita no satisface en algunas ocasiones todas sus expectativas. Ello se debe a la inestabilidad de la austenita cuando se presenta en la microestructura en forma de grandes bloques atrapados entre los haces de bainita. [14]-[18].

\section{PROCESO DE ADQUISICIÓN DE LA BAINITA}

El austenizado es un proceso de tratamiento térmico desarrollado para obtener una estructura que es $100 \%$ bainita. Este se logra calentando primero la pieza de la temperatura de austenización propia, seguida de un rápido enfriamiento en un baño de sal mantenido en el intervalo de bainita (generalmente entre 400 y $800^{\circ} \mathrm{F}$ ). La pieza se deja en el baño hasta que se completa la transformación a bainita.

La comparación de las propiedades mecánicas desarrolladas por el austenizado y por el método de temple y revenido suele hacerse a la misma dureza o resistencia. La superioridad del austenizado destaca en tales propiedades como reducción de área en tensión, resistencia al impacto y la prueba del combado lento. [19].
Fig. 4. TENACIDAD Y DUCTILIDAD MEJORADAS DE VARILLAS AUSTENIZADAS QUE SE COMPARAN CON VARILLAS TEMPLADAS Y REVENIDAS DE IGUAL DUREZA. [20]

DUREZA ROCKWELL C 50

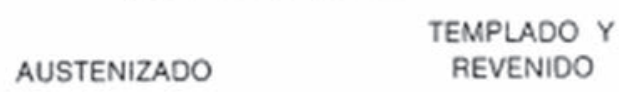

REDUCCION DEL AREA EN TENSION
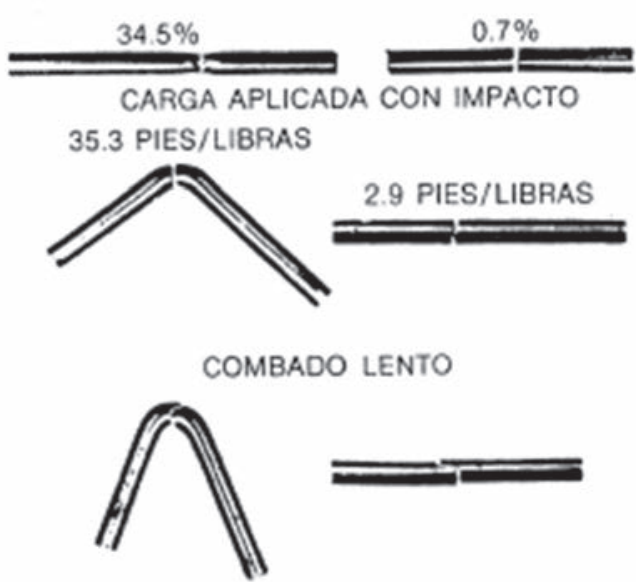

Fig. 5. COMPARACIÓN ENTRE EL MÉTODO DE AUSTENIZADO Y EL DE TEMPLADO Y REVENIDO PARA UN ACERO [21]

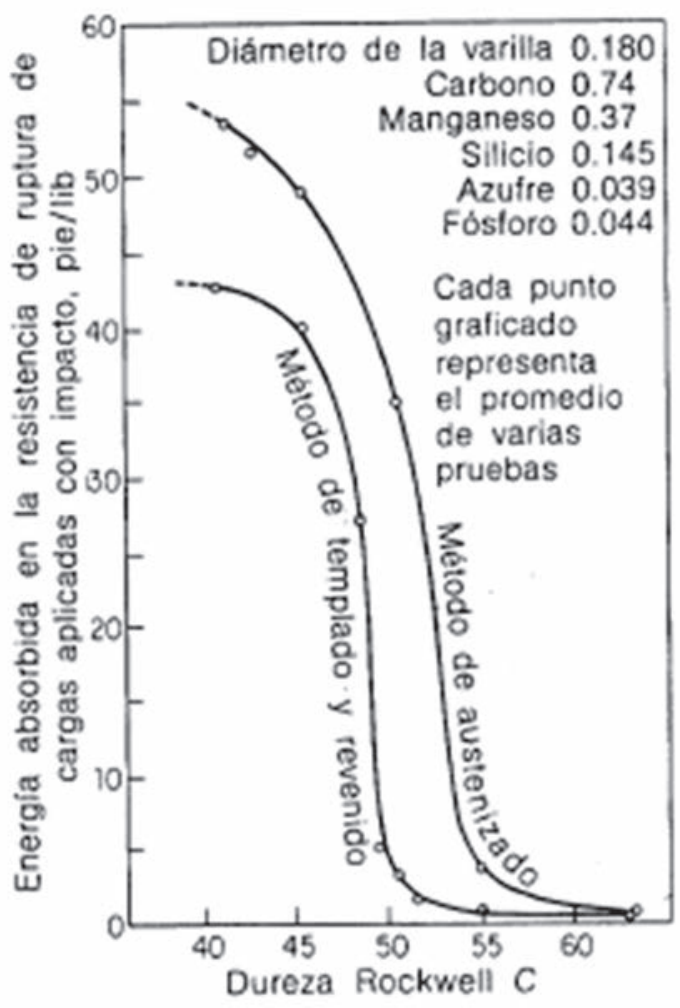

Este proceso tiene un inconveniente y es que son ideales las secciones que pueden enfriarse con suficiente rapidez para evitar la transforma- 
ción a perlita, en el intervalo de temperatura de 900 a $1200^{\circ} \mathrm{F}$; por tanto, la mayoría de las aplicaciones industriales han sido secciones de espesor menor a $1 / 2$ pulgada que puede incrementarse un poco con el empleo de aceros aleados, pero entonces el tiempo para terminar la transformación a bainita puede ser excesivo.

En los casos de la martensíta o de la ferrita Widmanstätten, y debido al bajo coeficiente de difusión del hierro a las temperaturas de la transformación bainítica, la deformación involucra un movimiento coordinado de átomos que, mediante una deformación homogénea, conforman la nueva red, existiendo una correspondencia de red entre la nueva fase y la fase de origen. De acuerdo con esto, mediante técnicas de alta resolución. [16]-[22]-[23]. Se ha mostrado que los átomos sustitucionales como $\mathrm{Mn}, \mathrm{Si}, \mathrm{Ni}$, Mo y $\mathrm{Cr}$ quedan "congelados" en sus posiciones originales tras la transformación bainítica.

\section{PROPIEDADES MECÁNICAS}

En cuanto a la fluencia, la bainita presenta un mejor comportamiento que la perlita y la ferrita proeutectoide y es superada por la martensíta. Sin embargo, debido a que la martensíta generalmente se utiliza en estado revenido, estas dos microestructuras pueden ser similares con respecto a la resistencia a la fluencia. Aún mas, cuando se obtiene bainita de baja temperatura, de $120^{\circ} \mathrm{C}$ a $200^{\circ} \mathrm{C}$, el espesor de las placas llega a ser tan pequeño, que la mezcla de esta bainita con la austenita retenida puede proporcionar durezas de 600 HV, como fue mostrado por algunos autores. [24].

Si tenemos en cuenta que a la ductilidad se le sumaría la energía que puede soportar al impacto, la bainita presenta un mejor comportamiento con respecto a la perlita y a la martensíta revenida, gracias al tamaño de las placas bainíticas y al menor tamaño de los carburos que presenta con respecto a la martensíta revenida. [25].

En la bainita hay que destacar que la inferior (baja temperatura en la fundición nodular) presenta una mayor tenacidad que la bainita superior (alta temperatura), a pesar de que también tiene una mayor resistencia a la fluencia. Esto puede ser atribuido al menor tamaño de los paquetes y de las placas, los cuales tienen un cambio mayor en la orientación entre sus límites que incrementa el obstáculo tanto al movimiento de las dislocaciones como a la propagación de las grietas. [26].

Se debe tomar en cuenta si la austenita está en forma de películas o bloques; la austenita en bloques afecta desfavorablemente la resistencia a la fluencia y la tenacidad, mientras que en forma de películas mejora el comportamiento en estas mismas propiedades. [27]. Al contar con un adecuado control del tamaño de grano austenítico y del ancho de los paquetes de subunidades, incluso la bainita superior podría presentar una mayor tenacidad que la martensíta revenida del mismo nivel de resistencia. [28].

\section{ACEROS BAINÍTICOS Y ESTUDIOS SOBRE LA INFLUENCIA DEL BORO}

El boro ha sido utilizado como elemento aleante que aumenta la templabilidad en aceros de baja aleación y, a su vez, cuando se agrega en cantidades sumamente pequeñas, que al ser comparadas con otros elementos aleantes serían despreciables, incrementa de forma considerable la dureza de los aceros. López - Chipres [37] en su escrito, dice que según la naturaleza dual del átomo de boro en la austenita y su entorno, este se puede encontrar en posición sustitucional o intersticial, si el átomo tiene suficiente energía, puede pasar de una posición sustitucional a una intersticial con lo que podrá difundir más rápidamente; esta teoría es complementada por los estudios realizados por Wang [38], los cuales hablan de difusión intersticial del boro porque los resultados obtenidos con este elemento se pueden asemejar a los resultados obtenidos en la difusión del carbono.

El principal efecto del boro es la inhibición de la ferrita en el límite de grano austenítico, pues facilita la formación de bainita y martensíta y proporciona de esta forma la templabilidad del acero, este efecto es debido al hecho que la segregación del boro hacia los límites de grano de la austenita disminuye la energía interfacial al reducir los sitios preferenciales para la nucleación de la ferrita [39], de todas formas si la cantidad de boro es muy alta se supera fácilmente el límite de solubilidad con lo cual se formarían boruros en los límites de grano que favorecen la nucleación de ferrita [40]. 
La segregación del Boro hacia los límites de grano de la austenita en los aceros bainíticos modifica sus características termodinámicas y da lugar a un ablandamiento de la austenita a trabajar en caliente [41]. Estudios realizados por Stumpf [42] y Song [43] sobre la fluencia en caliente en aceros, muestran que el pico de tensión y el pico de deformación en las curvas de fluencia tiene tendencia a disminuir a medida que se aumenta el contenido de boro.

Hay estudios en los cuales se hacen comparaciones de los aceros bainíticos aleados con boro respecto a los aceros perlíticos, en los cuales se muestra cómo varían las propiedades mecánicas como la resistencia ultima y el esfuerzo de fluencia entre otros. La Tabla I muestra un incremento de $399 \mathrm{MPa}$ (36\%) en la resistencia última, $485 \mathrm{MPa}(77 \%)$ de incremento en el esfuerzo de fluencia y $1.7 \%$ (15\% relativo) en la deformación, ubicando a los aceros bainíticos en un mayor escalafón respecto a los aceros perlíticos, dándoles muchos campos de aplicación.

TABLA I.

TABLA COMPARATIVA DE PROPIEDADES MECÁNICAS RELEVANTES ENTRE ACERO PERLÍTICO CONTRA ACERO BAINÍTICO J6 [44]

\begin{tabular}{|c|c|c|}
\hline $\begin{array}{c}\text { Propiedades } \\
\text { mecanicas }\end{array}$ & Acero perlitico & Acero Bainitico J6 \\
\hline $\begin{array}{c}\text { Esfuerzo Último } \\
\text { (MPa) }\end{array}$ & 1114 & 1513 \\
\hline $\begin{array}{c}\text { Esfuerzo de Fluen- } \\
\text { cia (MPa) }\end{array}$ & 624 & 1109 \\
\hline Elongación (100\%) & 11.1 & 12.8 \\
\hline $\begin{array}{c}\text { Tenacidad de Frac- } \\
\text { tura Ki (MPa) }\end{array}$ & 95 & 150 \\
\hline
\end{tabular}

Fig. 6. CURVA ESFUERZO VS. DEFORMACIÓN DE UN ACERO BAINÍTICO ALEADO CON BORO (LÍNEA ROJA) Y UN ACERO PERLITICO (LÍNEA AZUL) [44]

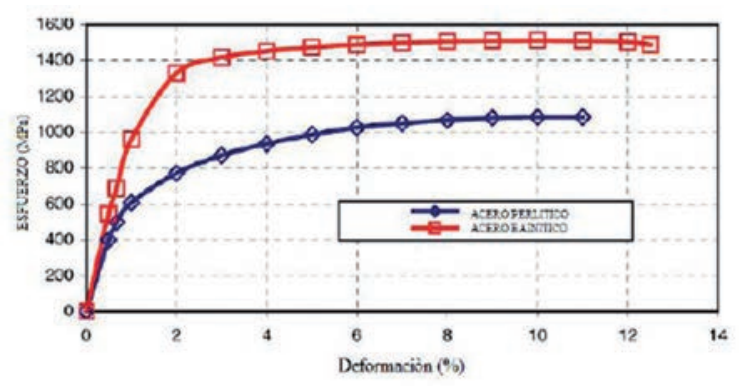

Miihkinen y Edmonds [50]-[51] realizaron investigaciones sobre dos aceros bainíticos con alto contenido en Silicio, de composición nóminas Fe0,2C-2Si-3Mn y Fe-0,4C-2Si-4Ni en porcentaje en peso, ambas aleaciones presentaban niveles de resistencia y tenacidad cercano a los de los aceros comerciales martensíticos de alta resistencia cuando se trataban isotérmicamente a las temperaturas de transformación bainítica. Aunque este tipo de aleaciones se hicieron con fines netamente académicos y no con algún enfoque industrial.

Hay otros trabajos en los cuales se ha variado los porcentajes de boro dentro de aceros bainíticos, analizan cambios relevantes en las propiedades mecánicas, este es el caso de Dian [45], el cual evalúa el crecimiento del esfuerzo de fluencia, el esfuerzo último y la elongación y los cuales son notorios en las Figuras 7,8 y 9

Fig 7,8 y 9. VARIACIÓN DE LAS PROPIEDADES MECÁNICAS CON RESPECTO A LOS CONTENIDOS DE BORO [45]
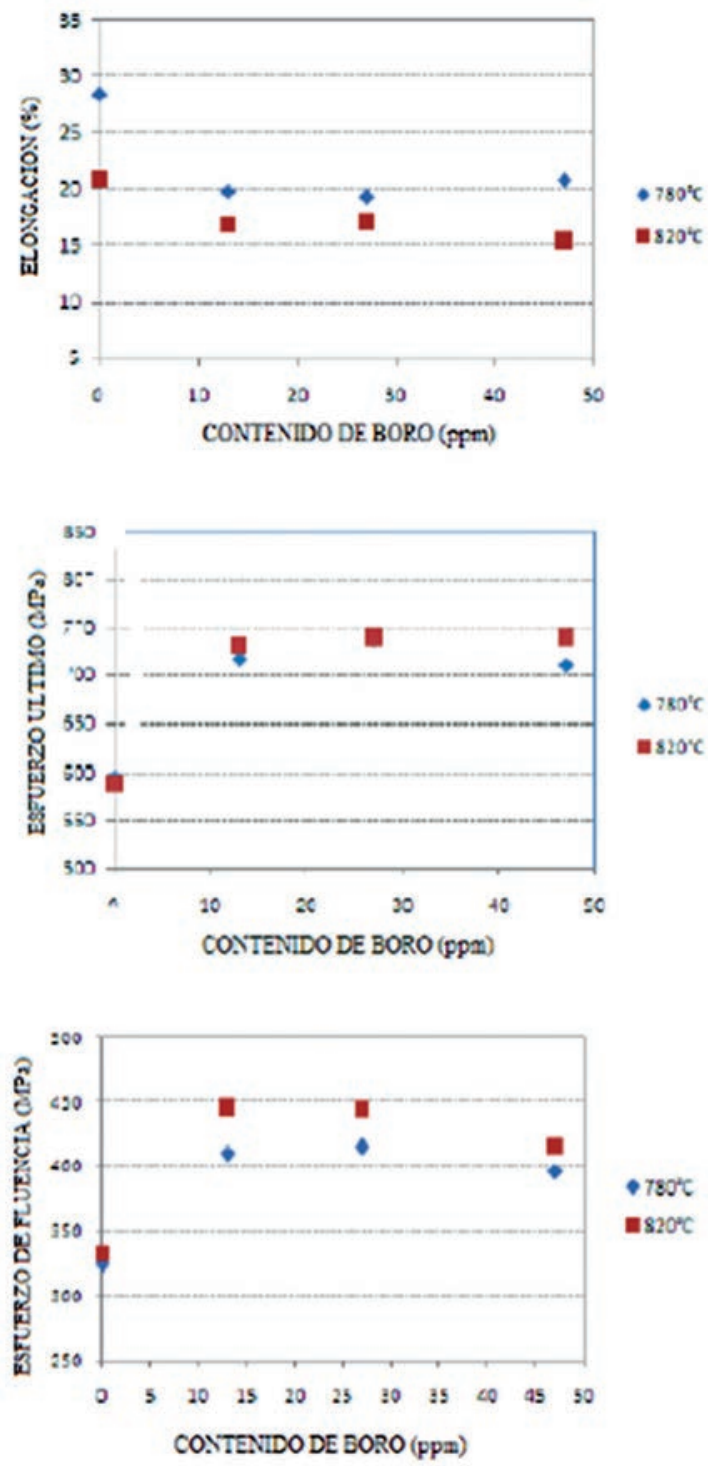
Otro trabajo que vale la pena mencionar es el realizado por Bedolla [46], en el cual analiza la variación de las propiedades mecánicas de aceros aleados con boro respecto a dos medios de enfriamiento (aire y agua), en este trabajo muestra que las estructuras bainíticas fueron generadas a raíz del enfriamiento al aire después del proceso de laminación, mientras que en el acero enfriado en agua predominó la presencia de martensita, por otro lado, el boro mostró un efecto favorable en la formación de bainita superior y se concluyó que la mejor combinación de propiedades mecánicas se obtuvo cuando el acero presentó 76 ppm de boro y un enfriamiento al aire, el cual obtuvo valores de esfuerzo máximo de 830MPa, esfuerzo de fluencia de $750 \mathrm{MPa}, 15 \%$ de elongación y una dureza promedio de 4OHRc.

Fig 10 y 11. PROPIEDADES MECÁNICAS DEL ACERO ENFRIADO AL AIRE Y ENFRIADO EN AGUA EN FUNCIÓN DEL CONTENIDO DE BORO [46]
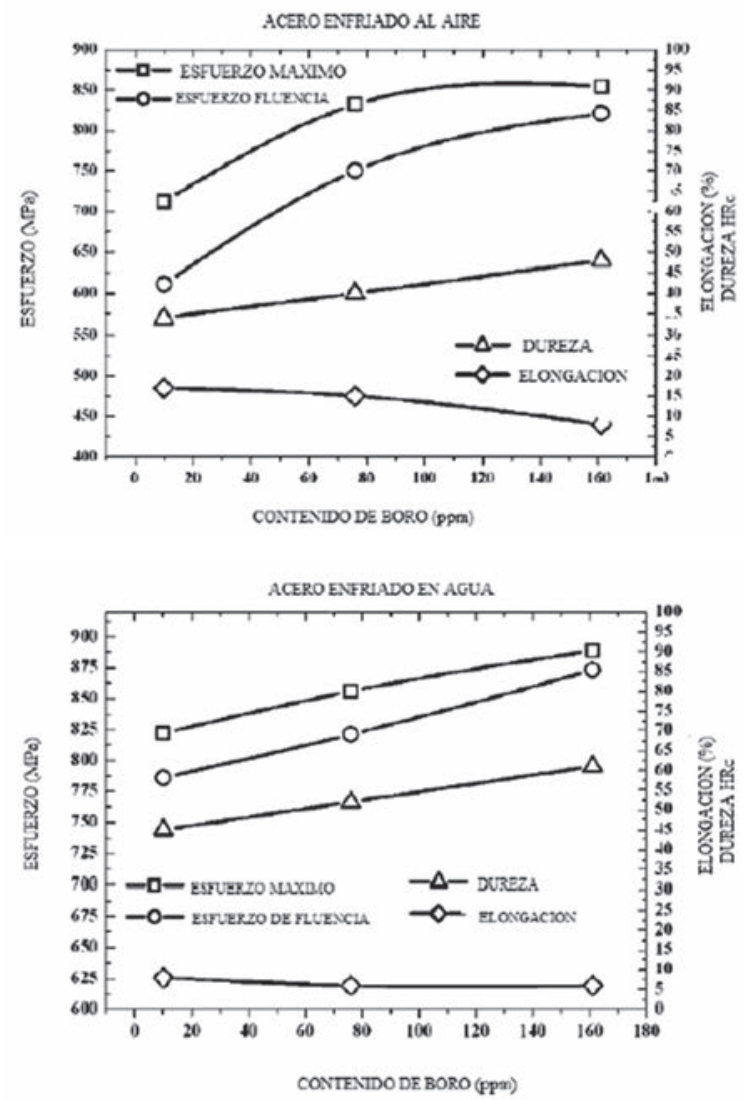

En los últimos tiempos ha surgido una nueva clase de aceros bainíticos libres de carburos, conocidos como aceros nanoestructurados, formados a bajas temperaturas de transformación de bainita, para obtener materiales con subunidades de bainita de 20 a 100nm de espesor [29]. Con el desarrollo de estos nuevos aceros se pueden alcanzar valores en la energía de impacto Charpy entre 24-44 J, además, en ensayos de tensión se obtienen esfuerzos entre 1600 a $1800 \mathrm{Mpa}$. [26]. La nanoestructura bainítica es diseñada para evitar la formación de cementíta, la cual es muy estable debido a las adiciones de manganeso [30].

En la actualidad aún se hacen estudios sobre aceros superbainíticos, nuevas estructuras y mejora de propiedades mecánicas por medio de tecnologías de punta aplicadas a este tipo de materiales, es el caso de la investigación de un grupo de posdoctores en la Universidad de Cambridge. Se han desarrollado aceros bainíticos libres de carburos y altos en carbono $(0.8 \%)$ que por mantenimiento isotérmico a temperaturas muy bajas $\left(125-300^{\circ} \mathrm{C}\right)$ durante periodos de tiempo razonables a escala industrial, dan lugar a una estructura mixta de ferrita-bainítica, excepcionalmente fina (20-80nm), y austenita retenida. La finura de la microestructura junto con la alta fracción de ferrita-bainítica presente $(\approx 80 \%)$ confieren al material de unas propiedades mecánicas nunca antes conseguidas en aceros de este tipo. Esfuerzo de fluencia $=2.5 \mathrm{GPa}, \mathrm{KIC}=45 \mathrm{MPa} \sqrt{\mathrm{m}}, \mathrm{HV} 30=650$. En la actualidad, estos aceros se utilizan para la fabricación de blindajes, rodamientos y componentes de cajas de cambios sometidos a grandes esfuerzos [47]-[48]-[49].

\section{REFERENCIAS}

[1] H. K. D. H. Bhadeshia. Bainite in Steels: Transformations, Micostructure and Poperties - 2nd Edition. Published by the Institute of Materials. Publication Date March 2001.

[2] http://www.zerneri.com/espanol/aboro.htm.

[3] Cristian Viáfara y Juan Manuel Vélez. Transformación Bainítica en aleaciones Fe-C. Ingeniería y Ciencia, ISSN. Septiembre de 2005.

[4] H.K.D.H. Bhadeshia. Bainite in Steels: Transformations, Microstructure and Properties, The Institute of Materials, 16-199 (1992).

[5] C.C. Viáfara, M.I. Castro, J.M. Vélez y A. Toro. Unlubricated Sliding Wear of Pearlitic and Bainitic Steels, Wear, 259, 411-416 (2005). 
[6] F.B. Pickering. The estructure and Properties of Bainite in Steels, Symposium: Transformation and Hardenability in Steels, 1967.

[7] Bramfitt B L, Speer J G. Steels Alloys with Lower Bainite Microstructures for Use in Railroad Cars and Track. Federal Railroad Administration. 2002.

[8] Mauricio Alejandro Sierra Cetina. Obtención y evaluación de las propiedades mecánicas del acero bainítico Fe-0.32C-1.45Si- 1.97Mn1.26Cr-0.26Mo-0.10V aleado con Boro. BOGOTÁ, D.C. 2011.

[9] H K D H Bhadeshia, D V Edmonds. Metals Science. 17 (1983) 411-419.

[10] CHESTER, N. A. y BHADESHIA, H. K. D. H. (1997) Phys. IV France 7, Colloque C5: C5-41 - C5-46.

[11] CHANG, L. C. y BHADESHIA, H. K. D. H. (1995) Mat. Sci. and Tech., 11: 874-881.

[12] PARKER, S. V. (1997) Tesis Doctoral. Universidad de Cambridge.

[13] SINGH, S. B. y BHADESHIA, H. K. D. H. (1998) Mat. Sci. and Eng. A245: 72-79.

[14] H K D H Bhadeshia, D V Edmonds. Metals Science. 17 (1983) 411-419.

[15] H K D H Bhadeshia, D V Edmonds. Metals Science. 17 (1983) 420-425.

[16] H K D H Bhadeshia, A R Waugh. Acta Metals. 30 (1982) 775-784.

[17] S.J. Matas y R.F. Hehemann, Trans. Metall. Soc. AIME 221 (1961).] [V.T.T. Mihkinen y D.V. Edmonds, Mater. Sci. Technol. 3 (1987) 411-449.

[18] H.K.D.H. Bhadeshia, Mater. Sci. Technol. 15 (1999)

[19] Sydney H. Avner. Introducción a la Metalurgia Física. Segunda Edición. McGraw Hill. México. 1988.

[20] Research Laboratory, U.S. Steel Corporation.

[21] Suiting the heat treatment to the job; a simple discussion of the problems of heat treatment. Carnegie-Illinois Steel Corporation. United States Steel. 1946.

[22] STARK, I., SMITH, G. D. W. y BHADESHIA, H. K. D. H. (1990) Met. Trans A, Vol 21: 837-844.
[23] STARK, I., SMITH, G. D. W. y BHADESHIA, H. K. D. H. (1987) Phase Transformations' 87. Ed. por G. W. Lorimer., The Institute of Metals, Cambridge: 211- 215

[24] C. García-Mateo, F.G, Caballero y H.K.D.H. Bhadeshia. Low Temperature Bainite, International Conference on Martensitic Transformations (ICOMAT '02), Helsinki, Finland, 2002.

[25] H. Sierra. Aceros de Construcción Mecánica, de Herramientas e Inoxidables y Pautas para su selección, Universidad Nacional de Colombia, Medellín, 110-125 (2000).

[26] F.B. Pickering. Th estructure and Properties of Bainite in Steels, Symposium: Transformation and Hardenability in Steels, 1967.

[27] B.J.P. Sandvik y H.P. Nevalainen. StructureProperty Relationships in Commercial Low-Alloy Bainitic-Austenitic Steel With High Strength, Met. June, 213-20 (1981).

[28] J.P. Naylor y P.R. Krahe. The effect Of the Bainite Packet Size on Toughness, Met. Trans. 5, 16991701 (1974).

[29] M.N. Yoozbashi, S. Yazdani. Mechanical properties of nanostructured, low temperature bainitic steel designed using a thermodynamic model. Materials Science and Engineering A 527 [2010] 3200-3205.

[30] C.M. Amey, H. Huang, P.E.J. Rivera-Díaz-del-Castillo, Distortion in 100Cr6 and nanostructured bainite, Materials and Design 35 [2012] 66-71.

[31] F.C. Zhang, T.S. Wang, P. Zhang, C.L. Zheng, B. Lv, M. Zhang and Y.Z. Zheng. A novel method for the development of a low-temperature bainitic microstructure in the surface layer of low-carbon steel. Scripta Materialia 59 [2008] 294-296.

[32] Lihe Qian, Qian Zhou, Fucheng Zhang, Jiangying Meng, Ming Zhang, Yuan Tian. Microstructure and mechanical properties of a low carbon carbide-free bainitic steel co-alloyed with $\mathrm{Al}$ and Si. Materials and Design 39 [2012] 264-268.

[33] I. Mejía, A. Bedolla-Jacuindea, C. Maldonado, J.M. Cabrerab,c. Hot ductility behavior of a low carbon advanced high strength steel [AHSS] microalloyed with boron. Materials Science and Engineering A 528 [2011] 4468-4474.

[34] WANG Yong-wei , FENG Chun, XU Feng-yun , BAI Bing-zhe , FANG Hong-sheng. Influence of $\mathrm{Nb}$ on 
Microstructure and Property of Low-Carbon MnSeries Air-Cooled Bainitic Steel. JOURNAL OF IRON AND STEEL RESEARCH, INTERNXfIONAL. 2010, 17[1]: 49-53.

[35] ZHANG Han. BAl Bing-zhe, FANG Hong-sheng. A Mn-Series of Oil-Quenched Super-Strength Bainitic Steel With High Hardenability. JOURNAL OF IRON AND STEEL RESEARCH, INTERNATIONAL. 2010. 17[5]: 38-43

[36] M. Naderia, M. Ketabchia, M. Abbasia, W. Bleck. Analysis of microstructure and mechanical properties of different boron and non-boron alloyed steels after being hot stamped. Procedia Engineering 10 [2011] 460-465.

[37] LÓPEZ-CHIPRES, E. [et al.] Hot flow behavior of boron microalloyed steels. Materials Science and Engineering, 2008, págs. 49-55.

[38] WANG, W., ZHANG, S., HE, X. Diffusion of boron in alloys. Acta Metalurgia, Vol 43, 1994, pags. 1693-1699.

[39] BRAMFITT, BRUCE L., BENSCOTER, ARLON O. Metallographer's Guide. Practices and Procedures for Irons and Steels. ASM International, 2002.

[40] PORTER, D.A., EASTERLING, K.E. Phase Transformations in Metals and Alloys. Nelson Thorners, 1992.

[41] BRAMFITT, B.L., SPEER, J.G. A Perspective on the Morphology of Bainite. Metallurgical Transactions A, Vol. 21A, 1990, págs. 817-829.
[42] STUMPF, W., BANKS, K. The hot working characteristics of a boron bearing and a conventional low carbon steel. Materials Science and Engineering, 2006, pp. 86-94.

[43] SONG, S.H. Effect of Boron on the hot ductility of 2.25Cr1Mo steel. Materials Science and Engineering, 2003.

[44] AGLAN, H.A. Mechanical and fracture behavior of bainitic rail steels. Materials Process Technology. 2004, pags $268-274$.

[45] DIAN, F. Effect of boron on the microstructure and mechanics properties of cold rolled multiphase steels. 2011.

[46] BEDOLLA, J.A. High resistance boron treated steels for railways applications. Materials Science and Technology. 2009. Vol. 25. No. 3.

[47] C Garcia - Mateo, H K D H Bhadeshia, Material Science and Engineering. 378 (2004) 289 292.

[48] F.G. Caballero, H.K.D.H. Bhadeshia, K.J.A. MaweIla, D.G. Jones y P. Brown. Mater. Scien. Tech.18 (2002) 279.

[49] C. García-Mateo, F.G. Caballero , H. K. D. H. Bhadeshia. ISIJ Int. 43 (2003). 1238.

[50] V.T.T, Miihkinen y D.V. Edmonds, Mater. Sci. Technol. 3 (1987) 422-431.

[51] V.T.T, Miihkinen y D.V. Edmonds, Mater. Sci. Technol. 3 (1987) 432-440. 